\title{
Statistical Physics Approach to the Optimal Transport Problem
}

\author{
Patrice Koehl, ${ }^{1}$ Marc Delarue, ${ }^{2}$ and Henri Orland ${ }^{3}$ \\ ${ }^{1}$ Department of Computer Science and Genome Center, University of California, Davis, California 95616, USA \\ ${ }^{2}$ Unité de Dynamique Structurale des Macromolécules, Department of Structural Biology and Chemistry, \\ UMR 3528 du CNRS, Institut Pasteur, 75015 Paris, France \\ ${ }^{3}$ Institut de Physique Théorique, CEA-Saclay, 91191 Gif/Yvette Cedex, France
}

(Received 29 April 2019; revised manuscript received 3 June 2019; published 26 July 2019)

\begin{abstract}
Originally defined for the optimal allocation of resources, optimal transport (OT) has found many theoretical and practical applications in multiple domains of science and physics. In this Letter we develop a new method for solving the discrete version of this problem using techniques derived from statistical physics. We derive a strongly concave free energy function that captures the constraints of the OT problem at a finite temperature. Its maximum defines an optimal transport plan, or registration between the two discrete probability measures that are compared, as well as a pseudodistance between those measures that satisfies the triangular inequalities. The computation of this pseudodistance is fast and numerically stable. The temperature dependent OT pseudodistance is shown to decrease monotonically with respect to the inverse of the temperature and to converge to the standard OT distance at zero temperature, providing a robust framework for temperature annealing. We illustrate applications of this framework to the problem of image comparison.
\end{abstract}

DOI: 10.1103/PhysRevLett.123.040603

Imagine that there are $N_{1}$ flour milling plants around Paris, France, that serve $N_{2}$ bakeries within Paris, and let us assume balance, namely that there is as much flour produced by the plants as needed by the bakeries. A company in charge of the distribution of the flour will take into account the individual cost of transporting some amount of flour from one plant to one bakery to find an "optimal transport plan," namely an assignment of how much flour needs to be transported between each plant and each bakery that leads to a minimal overall cost for the transport. Finding a solution to this seemingly simple practical problem has led to the development of a small gem in the mathematics and statistics communities, namely the optimal transport (OT) problem. What makes the OT problem so interesting is that its solution includes two essential components. First, it defines a distance between the distributions considered, with such distance being referred to as the Monge-Kantorovich distance, the Wasserstein distance, or the earth mover's distance, depending on the field of applications. These distances have enabled statisticians and mathematicians to derive a geometric structure on the space of probability distributions $[1,2]$. Second, it also provides the optimal transportation plan between the distributions; this optimal plan defines a registration, thereby enabling alignment between the distributions. Applications of OT have exploded in the recent years, in domains such as machine learning, computer vision, and linguistics. Multiple fields of physics are also impacted, from applications of OT to density functional theory [3], quantum mechanics [4], stochastic thermodynamics [5], and general relativity [6], among others. For extensive reviews of OT and its applications, see $[1,2]$.

Our focus in this Letter is on the discrete version of the OT problem. We consider two sets of points $S_{1}$ of size $N_{1}$ and $S_{2}$ of size $N_{2}$. Each point $k$ in $S_{1}\left(S_{2}\right)$ is assigned a "mass" $m_{1}(k)\left[m_{2}(k)\right]$. The balance condition implies that $\sum_{k} m_{1}(k)=\sum_{l} m_{2}(l)$. We assume that these two sums are equal to 1 . We encode the cost of transport between $S_{1}$ and $S_{2}$ as a positive matrix $C_{k l}$ with $k \in\left\{1, \ldots, N_{1}\right\}$ and $l \in\left\{1, \ldots, N_{2}\right\}$. The OT problem can then be formulated as finding a matrix $G$ of correspondence between points in $S_{1}$ and points in $S_{2}$ that minimizes the transport cost $U$ defined as

$$
U(G)=\sum_{k, l} G_{k l} C_{k l}
$$

where the summations extend over all $k$ in $S_{1}$ and $l$ in $S_{2}$. The minimum of $U$ is to be found for the values of $G_{k l}$ that satisfy the following constraints:

$$
\begin{gathered}
\forall(k, l), \quad G_{k, l} \geq 0, \\
\forall k, \quad \sum_{l} G_{k l}=m_{1}(k), \\
\forall l, \quad \sum_{k} G_{k l}=m_{2}(l) .
\end{gathered}
$$


The solution to the OT problem provides an optimal transport plan $G_{\text {opt }}$ and the corresponding minimum transport cost $U_{\min }=U\left(G_{\mathrm{opt}}\right)$. The minimum cost defines a "distance" between the two sets of points. It has all the properties of a metric when the cost matrix $C$ is a metric matrix, see [1]. This metric is often referred to as the Wasserstein distance $W\left(S_{1}, S_{2}\right)$ between the two sets of points. In this Letter we will make the same assumption that $C$ is a metric matrix.

Note that the first condition on $G$, (2), extends to $0 \leq$ $G_{k l} \leq 1$ for all $k$ and $l$, based on our assumption that the sum of the discrete probability measures are 1 on both sets of points. Optimizing (1) under the constraints (2) is a linear programming (LP) problem. While much progress has been achieved for solving those problems [7], current practical implementations are roughly of order $O\left(n^{3}\right)$ [where $n=\max \left(N_{1}, N_{2}\right)$ ], with a quadratic complexity in the number of variables considered. Such complexity levels are considered problematic when $n$ is larger than a few thousands.

Interestingly, the current successes of OT have been triggered by the idea of minimizing a regularized version of Eq. (1):

$$
U_{\epsilon}(G)=\sum_{k l} G_{k l} C_{k l}-\epsilon \sum_{k, l} G_{k l} \ln \left(G_{k l}\right),
$$

where $\epsilon$ is the regularization parameter, and the second term is an entropic barrier that enforces the positivity of the $G_{k l}$ terms [8]. This regularized version of optimal transport is often called the Schrödinger problem [9]. It maps to the traditional OT problem as $\epsilon \rightarrow 0$; in addition, the optimal solution $U\left(G_{\text {opt }}\right)$ where $G_{\text {opt }}$ has been computed at a given $\epsilon$ is a pseudodistance, referred to as the Sinkhorn distance [8]. Note that it is labeled as a pseudodistance as it only satisfies the symmetry and triangular inequality properties of a metric [10]. The entropic penalization has the advantage that it defines a strongly convex problem with a unique solution [8]. Another advantage of the regularized OT problem is that its solution can be found efficiently using Sinkhorn's algorithm [11,12], with a time complexity of $O\left(n^{2}\right)$. This algorithm finds solutions for a given value of the relaxation parameter $\epsilon$. For small values of this parameter, numerical issues may arise and a stabilization is necessary [13]. Convergence of a stabilized Sinkhorn algorithm can nevertheless be very slow when $\epsilon$ is small. Such small values are, however, desirable for finding good approximations to the solution of the nonregularized OT problem. A popular heuristic solution to this problem is the so-called $\epsilon$ scaling, where one solves the regularized problem with gradually decreasing values for $\epsilon$ [14]. To our knowledge, no quantitative analyses of the convergence of such an $\epsilon$-scaling method are available.

In this Letter, we develop an alternate framework for solving the OT problem that is derived from a statistical physics point of view and report on its applications to computing the similarities between images. A more detailed theoretical and numerical analysis is presented in a companion paper [15]. In this framework, we exploit the formal analogy of the cost function in Eq. (3) to a free energy ( $\epsilon$ is then the analog of a temperature, $T$ ). It can be seen as a generalization of the so-called invisible hand algorithm, which used a similar approach for solving the assignment problem [16].

In statistical physics, a system that is in thermal equilibrium at finite temperature will sample many states. The corresponding Gibbs distribution represents the probability of this system to exist in any specific state. The most probable state is then the one with lowest energy. Hence, minimizing an energy function can be reformulated as the problem of finding the most probable state of the system it defines. Let us consider two sets of weighted points, a source set $S_{1}$ and a target set $S_{2}$ equipped with masses $\mathbf{m}_{1}$ and $\mathbf{m}_{2}$, respectively. To solve the OT problem between those two sets, the "system" is identified with the different transport plans between $S_{1}$ and $S_{2}$ that satisfy the constraints of mass balance and positivity. Those plans belong to a convex polytope denoted as $\mathcal{G}\left(S_{1}, S_{2}\right)$. Each state in this system is identified with a transport plan $G \in \mathcal{G}\left(S_{1}, S_{2}\right)$, and its corresponding energy $U(G)$ is defined in Eq. (1). The probability $P(G)$ associated with a transport plan $G$ is derived a

$$
P(G)=\frac{1}{Z_{\beta}\left(S_{1}, S_{2}\right)} e^{-\beta U(G)} .
$$

In this equation, $\beta=1 /\left(k_{B} T\right)$ where $k_{B}$ is the Boltzmann constant and $T$ the temperature, and $Z_{\beta}\left(S_{1}, S_{2}\right)$ is the partition function computed over all states of the system. This partition function is given by

$Z_{\beta}\left(S_{1}, S_{2}\right)=e^{-\beta \mathcal{F}_{\beta}\left(S_{1}, S_{2}\right)}=\int_{G \in \mathcal{G}\left(S_{1}, S_{2}\right)} e^{-\beta U(G)} d \mu_{12}$

where $d \mu_{12}$ can be seen as the Lebesgue measure for the space of transport plans $\mathcal{G}\left(S_{1}, S_{2}\right)$ and $\mathcal{F}_{\beta}\left(S_{1}, S_{2}\right)$ is the free energy of the system. We note first two important properties of this free energy, namely that $\mathcal{F}_{\beta}\left(S_{1}, S_{2}\right)$ is symmetric and satisfies all triangle inequalities if the cost matrix $C$ is metric, and that it is a monotonically decreasing function of $\beta$ that converges to the traditional OT distance $W\left(S_{1}, S_{2}\right)$ (see the companion paper [15] for proofs). From those two properties, it is clear that this formulation of the optimal transport problem is appealing. It defines a temperature dependent free energy that satisfies metric properties when the cost function is metric, with a monotonic dependence on the temperature, and convergence to the actual OT distance at zero temperature. It is, unfortunately, of limited practical interest as the free energy cannot be computed 
explicitly. We propose a scheme for approximating the free energy using the saddle point approximation.

Taking into account the constraints defined in Eq. (2), the partition function can be written as

$$
\begin{aligned}
Z= & \int_{0}^{1} \prod_{k l} d G_{k l} e^{-\beta \sum_{k l} C_{k l} G_{k l}} \\
& \times \prod_{k} \delta\left(\sum_{l} G_{k l}-m_{1}(k)\right) \prod_{l} \delta\left(\sum_{k} G_{k l}-m_{2}(l)\right) .
\end{aligned}
$$

We use the Fourier representation of the delta functions, thereby introducing new auxiliary variables $\lambda_{k}$ and $\mu_{l}$, with $k \in\left\{1, \ldots, N_{1}\right\}$ and $l \in\left\{1, \ldots, N_{2}\right\}$, respectively. The partition function can then be written as (up to a multiplicative constant)

$$
\begin{aligned}
Z= & \int \prod_{k} d \lambda_{k} \int \prod_{l} d \mu_{l} \int_{0}^{1} \prod_{k, l} d G_{k l} \\
& \times e^{-\beta \sum_{k, l} G_{k l}\left(C_{k l}+i \lambda_{k}+i \mu_{l}\right)+\beta}\left(\sum_{k} i \lambda_{k} m_{1}(k)+\sum_{l} i \mu_{l} m_{2}(l)\right) .
\end{aligned}
$$

Performing the integration over the variables $G_{k l}$, we get,

$$
Z=\int \prod_{k} d \lambda_{k} \int \prod_{l} d \mu_{l} e^{-\beta F_{\mathrm{eff}}\left(\beta, i \lambda_{k}, i \mu_{l}\right)}
$$

where $F_{\text {eff }}$ is a functional, or effective free energy defined by

$$
\begin{aligned}
F_{\text {eff }}(\beta, \lambda, \mu)=- & \left(\sum_{k} \lambda_{k} m_{1}(k)+\sum_{l} \mu_{l} m_{2}(l)\right) \\
& -\frac{1}{\beta} \sum_{k l} \ln \left(\frac{1-e^{-\beta\left(C_{k l}+\lambda_{k}+\mu_{l}\right)}}{\beta\left(C_{k l}+\lambda_{k}+\mu_{l}\right)}\right) .
\end{aligned}
$$

Let $\bar{G}_{k l}$ be the expected value of $G_{k l}$ with respect to the Gibbs distribution given in Eq. (4). As mentioned above, it is unfortunately not possible to compute these expected values directly as the partition function defined in (8) is not known analytically. Instead, we derive a saddle point approximation (SPA) by looking for extrema of the effective free energy with respect to the variables $\lambda$ and $\mu$ :

$\frac{\partial \mathcal{F}_{\text {eff }}(\beta, i \lambda, i \mu)}{\partial \lambda_{k}}=0 \quad$ and $\quad \frac{\partial \mathcal{F}_{\text {eff }}(\beta, i \lambda, i \mu)}{\partial \mu_{l}}=0$.

After some rearrangements, those two equations can be written as

$$
\begin{aligned}
& \forall k, \quad \sum_{l} \bar{G}_{k l}=m_{1}(k), \\
& \forall l, \quad \sum_{k} \bar{G}_{k l}=m_{2}(l),
\end{aligned}
$$

where

$$
\bar{G}_{k l}=\phi\left[\beta\left(C_{k l}+i \lambda_{k}+i \mu_{l}\right)\right]
$$

and

$$
\phi(x)=\frac{e^{-x}}{e^{-x}-1}+\frac{1}{x} .
$$

As is often the case, the saddle point may be purely imaginary. In the present case, one can easily see from Eq. (11) that the variables $i \lambda_{k}$ and $i \mu_{l}$ must be real and in the following, we will replace $\left\{i \lambda_{k}, i \mu_{l}\right\}$ by $\left\{\lambda_{k}, \mu_{l}\right\}$. We observe also that Eqs. (9), (11), and (12) are invariant under the translation $\left\{\lambda_{k}+K, \mu_{l}-K\right\}$ where $K$ is an arbitrary constant. This translational degree of freedom leaves the free energy $F_{\text {eff }}$ unchanged.

To analyze the SPA, we need to check the existence and assess the unicity of the critical points of the free energy. In the companion paper [15], we have shown that the Hessian of $F_{\text {eff }}(\beta, \lambda, \mu)$ is negative semidefinite with $\left(N_{1}+N_{2}-1\right)$ strictly negative eigenvalues and one zero eigenvalue. Furthermore, the eigenvector corresponding to the zero eigenvalue is $(1, \ldots, 1,-1, \ldots-1)$ (with $N_{1} 1 s$, and $N_{2}-1 s$ ), and thus corresponds to the constant translation invariance of this free energy. Setting one of the parameters $\lambda_{k}$ or $\mu_{k}$ to zero, the free energy function on this restricted parameter space is strictly concave.

For a given value of $\beta$, the expected values $\bar{G}_{k l}$ form a transport plan $G_{\text {opt }}$ between $S_{1}$ and $S_{2}$ that is optimal with respect to the effective free energy. We can associate to this transport plan an optimum mean field energy $U_{\beta}^{M F}\left(G_{\mathrm{opt}}\right)$. This energy satisfies some important properties. Namely, for all $\beta>0$ and cost metric matrix $C, d_{\beta}\left(S_{1}, S_{2}\right)=$ $U_{\beta}^{M F}\left(G_{\text {opt }}\right)$ is a temperature dependent pseudodistance between $S_{1}$ and $S_{2}$ that satisfies the symmetry and triangular inequality properties of a metric [it is not a true distance as $d_{\beta}\left(S_{1}, S_{1}\right)>0$ for $\beta$ finite]. Furthermore, $d_{\beta}\left(S_{1}, S_{2}\right)$ is a monotonic decreasing function of the parameter $\beta$ that converges to the transport distance $W\left(S_{1}, S_{2}\right)$. The validity of those two assertions is proved in [15].

The properties of the free energy functional and of the optimized mean field energy of the system highlight a number of advantages of the proposed framework. First, at each temperature the OT problem is turned into a strongly concave problem with a unique solution. This problem has a linear complexity in the number of variables. The concavity allows for the use of simple algorithms for finding a maximum of the free energy functional [Eq. (9)]. We have used an iterative Newton method to solve those equations. In the companion paper [15], we have shown that these equations can be solved with a time complexity of $O\left(n^{2}\right)$, thereby making this approach competitive with the entropy regularized OT method. We note 


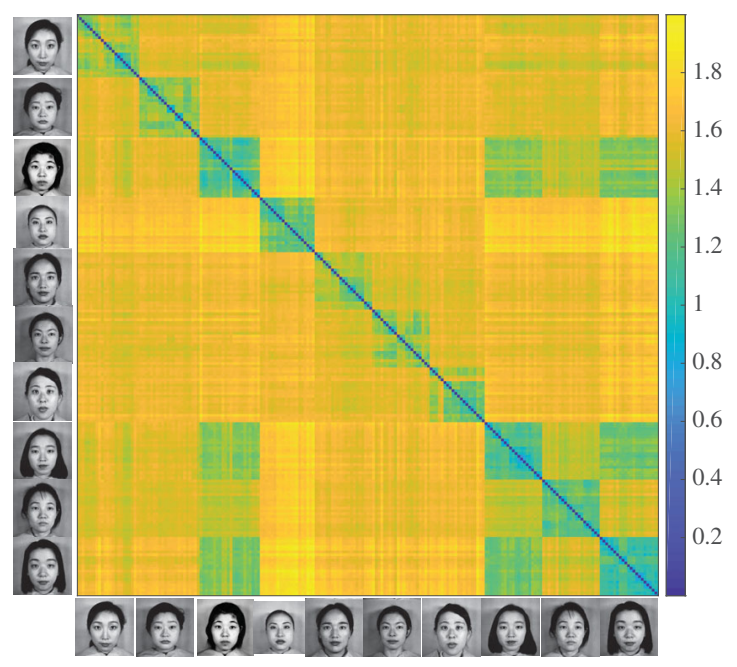

FIG. 1. Optimal transport distance matrix between all images in the JAFFE facial expression database. The distance $D(\infty)(k, l)$ is the converged transport energy between the SURF key points of the two images $k$ and $l$ (see text for details). The different images for the same model appear closer to each other than to images of other models. Interestingly $D(\infty)$ shows similarity between models 3,8 , and 10; those are the only 3 models whose hair covers their ears.

also that Eq. (12) has better numerical stability than the operations involved in the Sinkhorn algorithm, even at low temperatures, because of the ratio of exponentials in the definition of the function $\phi$. Second, the modified problem defines an optimal pseudodistance at each temperature that converges to the traditional OT distance when $T \rightarrow 0$. Finally, the convergence as a function of temperature is monotonic.

We present some computational examples that illustrate the use of our framework. We used the publicly available Japanese Female Facial Expression (JAFFE) database [17,18]. This database comprises 213 images of facial expressions posed by 10 Japanese female models. We refer to the images of one of the models as a class. Each class contains several different poses, namely 6 basic facial expressions +1 neutral. We characterized each image by selecting a set of "key points" using the SURF procedure. SURF is an image feature detector and descriptor used for object recognition and image registration [19]. Within SURF, a key point is a pixel within the image that is expected to be significant, i.e., a signature feature of the image. The significance is defined from a local neighborhood of the pixel of interest, characterized by a vector of 64 features. A pair of images is represented with their sets of key points, $S_{1}$ and $S_{2}$; the cost matrix $C$ between those key points, such that $C_{k l}$ between a key point $k$ on image 1 and a key point $l$ on image 2, is equal to the Euclidean distance between their feature vectors. The masses of the key points are set uniform. We computed a set of matrices $D(\beta)$ for $\beta$ ranging between 1000 and $10^{10}$, such that $D(\beta)(k, l)$ is the optimized transport energy $d_{\beta}\left(S_{k}, S_{l}\right)=U_{\beta}^{M F}\left(G_{\text {opt }}\right)$, i.e., the temperature-based pseudodistance between the sets of key points $S_{k}$ and $S_{l}$ of the images $k$ and $l$. We also computed $D(\infty)$, the matrix of distances at convergence. See Fig. 1 for a graphical representation of $D(\infty)$.

In order to assess the discriminative power contained in the different matrices $D(\beta)$, we considered a set of classification tasks as follows: we randomly selected half the images from each class to form a training set and used it for performing a 1-nearest neighbor classification (where nearest is with respect to the pseudodistance $d_{\beta}$ to the remaining images). By simple comparison between the class predicted by the classifier and the actual class to which the image belongs we obtain an estimate for the probability of correct classification $P(\beta)$ using $d_{\beta}$. We then repeat this procedure for 10000 random choices of the training set. In Fig. 2, we plot $P(\beta)$ as a function of $\beta$. Note that the lower the temperature (or alternatively the higher the parameter $\beta$ ), the more discriminative the pseudodistance $d_{\beta}$. Optimal classification (99.9\%), however, is already obtained for $\beta=10^{6}$, i.e., much before convergence to the optimal transport distance, usually reached for $\beta>10^{9}$. This high success rate should be compared to a success rate of $37 \%$ when the Hausdorff distance [20] is used to compare two images (using the same key point representation of the image, with the same Euclidean-based distance between key points).

We have claimed above that the temperature-based OT method enables a fast and robust solution to the OT problem. To check that it is indeed the case, we have compared our implementation of this method, FrEEOT, with our own implementation, ENTROPYOT, of the entropy

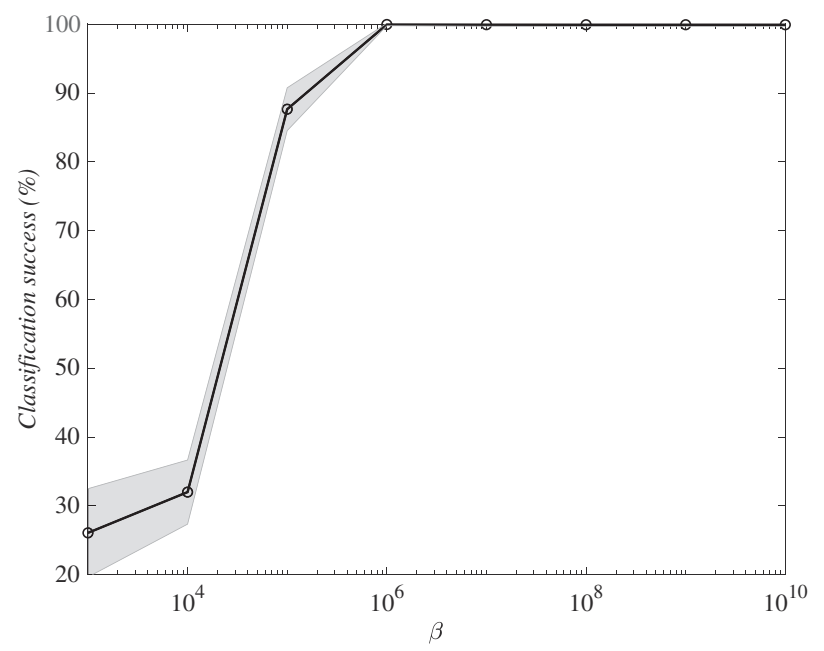

FIG. 2. Discriminative power of the temperature-based OT pseudodistances for images in the JAFFE database. The probability of correct classification using the pseudodistance measure $d_{\beta}$ (see text for details) is plotted against $\beta=1 / T$. The solid line corresponds to the arithmetic means computed over 10000 classification experiments (see text for details). Shaded areas represent standard deviations. 


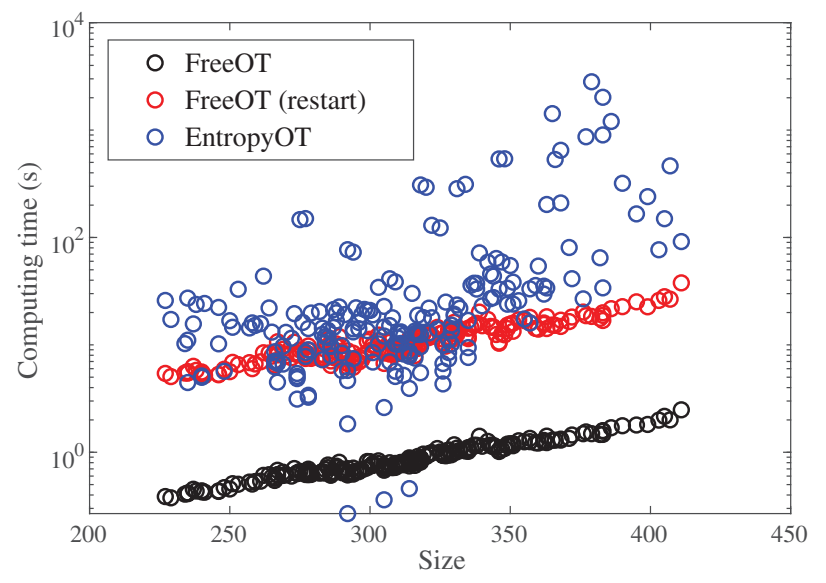

FIG. 3. The average running times for FREEOT without (black circles) and with reset (red circles) and for ENTROPYOT used for comparing two images is plotted against the number of key points in the images (see text for details).

regularized approach to the OT problem to compare images, as described above. ENTROPYOT is based on a log-domain stabilization and eta-scaling heuristic [14] and an overrelaxation scheme [21]; both modifications to the original algorithm of Cuturi [8] are expected to improve convergence and robustness. We have compared each image in the JAFFE dataset against five other images that have similar numbers of key points. Each comparison is performed until convergence, i.e., until the relative change in the energy falls below a tolerance of $10^{-6}$. Such convergence is usually reached for $\beta=10^{11}$ (or equivalently for $\epsilon=10^{-11}$ for ENTROPYOT). The computing times for FrEEOT and ENTROPYOT, averaged over the 5 comparisons, are plotted against the number of key points in the images in Fig. 3. With only a few exceptions, FREEOT is always found to be faster than ENTROPYOT, as the latter is found to slow down significantly for very small $\epsilon$ values. While convergence with high precision may not be needed, we observe that FREEOT is free of those convergence problems. Both FreEOT and ENTROPYOT include a scaling of the regularization parameter. For FREEOT, the values of the converged parameters $\lambda$ and $\boldsymbol{\mu}$ at one value of $\beta$ are used as input to the next value of $\beta$ considered. This is expected to improve convergence. To check if this is true, we repeated the calculations with FrEeOT by resetting $\lambda$ and $\boldsymbol{\mu}$ to zero for each $\beta$ value. The results are shown in Fig. 3, as FREEOT (reset). The reset does lead to less efficient convergence. Of significance, however, the computations remain feasible, even for very large $\beta$ values. In contrast, similar experiments with ENTROPYOT failed for most image comparisons, due to numerical instabilities for $\epsilon<10^{-5}$.

In summary, we have used statistical physics to derive an alternative representation of the discrete optimal transport problem. We have constructed a strongly concave effective free energy function that captures the constraints of the
OT problem. This effective free energy function is parametrized by temperature. Its maximum defines an optimal transport plan as well as a pseudodistance between the two sets of points considered. In the companion paper [15], we have shown that this formalism can be implemented in an algorithm with the same time complexity as those of the implementations of the regularized OT algorithms in time complexity, making it a competitive approach to solving the OT problem and therefore amenable to applications in data sciences. In addition, the temperature dependent OT pseudodistance is shown to decrease monotonically with respect to the parameter $\beta$ to the standard optimal transport or Wasserstein distance, thereby providing a robust framework for temperature annealing, a process that is still elusive for the entropy regularized optimal transport problem. Addressing issues of partial transport, as well as extensions to other transport problems such as the computation of the Gromov-Wasserstein distance [22] are promising directions for future work.

The work discussed here originated from a visit by P. K. at the Institut de Physique Théorique, CEA Saclay, France. He thanks them for their hospitality and financial support.

[1] C. Villani, Optimal Transport: Old and New, Grundlehren der mathematischen Wissenschaften (Springer, New York, 2008).

[2] G. Peyré and M. Cuturi, arXiv:1803.00567.

[3] G. Buttazzo, L. Pascale, and P. Gori-Giorgi, Phys. Rev. A 85, 062502 (2012).

[4] Y. Chen, T. T. Georgiou, and A. Tannenbaum, IEEE Trans. Autom. Control 63, 2612 (2018).

[5] E. Aurell, C. Mejía-Monasterio, and P. MuratoreGinanneschi, Phys. Rev. Lett. 106, 250601 (2011).

[6] A. Mondino and S. Suhr, arXiv:1810.13309.

[7] G. Sierksma and Y. Zwols, Linear and Integer Optimization: Theory and Practice, 3rd ed. (CRC Press, New York, 2015), http://www.taylorfrancis.com/books/ 9780429159961.

[8] M. Cuturi, in Advances in Neural Information Processing Systems 26, edited by C. J. C. Burges, L. Bottou, M. Welling, Z. Ghahramani, and K. Q. Weinberger (Curran Associates, Inc., New York, 2013), pp. 2292-2300.

[9] C. Léonard, Discrete Contin. Dyn. Syst. Ser. A 34, 1533 (2014).

[10] S.-I. Amari, R. Karakida, M. Oizumi, and M. Cuturi, Neural Comput. 31, 827 (2019).

[11] R. Sinkhorn, Ann. Math. Stat. 35, 876 (1964).

[12] R. Sinkhorn and P. Knopp, Pac. J. Math. 21, 343 (1967).

[13] L. Chizat, G. Peyré, B. Schmitzer, and F.-X. Vialard, Math. Comput. 87, 2563 (2018).

[14] B. Schmitzer, arXiv:1610.06519.

[15] P. Koehl, M. Delarue, and H. Orland, companion paper, Phys. Rev. E 100, 013310 (2019).

[16] J. Kosowsky and A. Yuille, Neural Netw. 7, 477 (1994).

[17] M. Lyons, S. Akamatsu, M. Kamachi, and J. Gyoba, in Proceedings of the Third IEEE International Conference on 
Automatic Face and Gesture Recognition (IEEE, New York, 1998), pp. 200-205, https://ieeexplore.ieee.org/document/ 670949.

[18] M. Lyons, J. Budynek, and S. Akamatsu, IEEE Trans. Pattern Anal. Mach. Intell. 21, 1357 (1999).

[19] H. Bay, A. Ess, T. Tuytelaars, and L. VanGool, Comput. Vision Image Understanding 110, 346 (2008).

[20] R. Rockafellar and R. J.-B. Wets, Variational Analysis (Springer-Verlag, Berlin, Heidelberg, 2005), http://www .springer.com/books/gp/9783540627722.
[21] A. Thibault, L. Chizat, C. Dossal, and N. Papadakis, in NIPS'17 Workshop on Optimal Transport \& Machine Learning, Long Beach, United States (2017) [arXiv:1711.01851].

[22] F. Mémoli, in Eurographics Symposium on Point-Based Graphics, edited by M. Botsch, R. Pajarola, B. Chen, and M. Zwicker (The Eurographics Association, 2007), pp. 81-90, http://dx.doi.org/10.2312/SPBG/SPBG07/ 081-090. 